\title{
Channel Aware Scheduling Algorithm for LTE Uplink and Downlink
}

\author{
Aswin Kanagasabai, Amiya Nayak \\ School of Electrical and Computer Engineering, \\ University of Ottawa, Canada \\ email:\{akana083,nayak\}@uottawa.ca
}

Received: August 30, 2015

Accepted: Nov. 3, 2015

Published: Nov. 28, 2015

DOI: 10.5296/npa.v7i3.8229

URL: http://dx.doi.org/10.5296/npa.v7i3.8229

\begin{abstract}
In the past two decades, there has been a drastic increase in the mobile traffic, which is caused by the improved user experience with smart phones and its applications. In LTE system, the packet scheduler plays a vital role in the effective utilization of the resources. This field is not standardized and has immense scope of improvement, allowing vendor-specific implementation. LTE scheduling can be categorized into two extremes, namely, Opportunistic scheduling and Fairness scheduling. The Best Channel Quality Indicator (BCQI) algorithm falls under the former category while Proportional Fairness (PF) algorithm under the later. BCQI algorithm provides high system throughput than PF algorithm, however, unlike BCQI algorithm, PF algorithm considers users with poor channel condition for allocation process. In this work, a new scheduling algorithm called as Opportunistic Dual Metric (ODM) Scheduling Algorithm is proposed for LTE uplink and downlink.
\end{abstract}

The objective of the algorithm is to prioritize the users with good channel condition for resource allocation, at the same time not to starve the users with poor channel conditions. The proposed algorithm has two resource allocation matrices, one being throughput-centric and the other being is fairness-centric. The uplink algorithm uses the two resource allocation matrices to allocate the resources to the users and to ensure contiguous resource allocation. The downlink algorithm is an extension of the proposed uplink algorithm avoiding uplink constraints. The downlink algorithm employs the two resource distribution matrices to provide an efficient resource allocation by expanding the allocation for the users considering intermittent resources. The performance of ODM is measured in terms of throughput, fairness. Additionally, the uplink algorithm is analyzed in terms of transmit power. From the results it is observed that the proposed algorithm has better trade-off in terms of all the performance parameters than PF scheduler and BCQI scheduler. 
Keywords: LTE Downlink, LTE scheduling, LTE Uplink, Opportunistic Proportional Fairness

\section{Introduction}

The evolution of the Third Generation Partnership Project Long Term Evolution (3GPP LTE) is a result of the growing need for improved performance in terms of factors such as data rate and latency improvements in the existing Third Generation (3G) system. LTE is predominantly accepted as a potential candidate for Fourth Generation (4G) system. The 3GPP introduced the LTE [1] in Release 8 [2] [3] to address the user demands such as high data rate and better quality of service. The LTE system supports diversified traffic of very high volume when compared to the Universal Mobile Telecommunications System (UMTS) proposed in 3GPP Release 5. The LTE system employs Radio Resource Management (RRM) performs functions such as link adaptation, Hybrid Automatic Repeat Request (HARQ) [12], resource scheduling, power control and Channel Quality Indicator (CQI) feedback to improve the resource utilization. Radio Resource Management (RRM) of LTE has been one of the active research topic in the recent years. One of the key components of RRM is the packet scheduler (PS). It plays a vital part in effective utilization of resources. PS manages the available resource and computes an optimal solution to allocate the available resources to users based on the various factors. The implementation of schedulers is vendor-specific and it is open to research. The objective of our work is to design new scheduling algorithms for uplink and downlink, to distribute the available resources among the active users, by exploiting their channel conditions. The channel conditions experienced by the users within the same cell vary with one another. This principle is used to prioritize the users with better channel conditions and thereby, achieving better spectral efficiency. At the same time, unfair allocation of resources is avoided by considering the allocation history of the users.

In order to distribute the resources effectively, many standard scheduling algorithms such as Best CQI (BCQI) algorithm and Proportional Fairness (PF) algorithms were proposed. BCQI algorithm prioritizes users with good channel condition, thereby improving the throughput of the system. While PF scheduling algorithm, which is one of the efficient algorithms, produces a trade-off between throughput performance as well as fairness in allocating resources to users by considering the users achieved throughput. Many variants of PF schedulers were proposed. The author in [20] proposed a Nash product based method to effectively allocate resources to users. The author in [16] proposed a modification by revising the metric after every transmission time interval (TTI) to improve fairness factor. In [21] the author has adopted a search-tree pattern for resource allocation. However, the allocation process is obsolete, as each user was allocated with only one resource. The author [15] proposes an uplink scheduling algorithm considering best effort and delay sensitive traffic. The decision metric, which is referred as reward function by the author, is computed specific to the type of traffic flow. In [17] the author proposes a scheme to increase the energy efficiency of the LTE system by proposing algorithm referred as Energy-Efficient Score 
Based Scheduler. The algorithm works under the principle of bandwidth trade-off. Norlund et al in [26] has proposed a new scheduling algorithm for wireless networks known as “Opportunistic Proportional Fairness” scheduling algorithm, combining the benefits of both PF and BCQI algorithms. Although the author has not tested the result in LTE networks, the idea behind combining PF and BCQI metrics can be adapted for LTE networks. The author in [22] proposed a similar idea for LTE downlink with the flexibility to choose between maximum data rate and PF metrics. However, [26] and [22] fail to provide the reasons behind choosing the co-efficients in the metric. Our work draws its motivation from [26] and proposes necessary modification to the metric by relaxing the assumptions made by the author.

In this work, a new LTE scheduling discipline referred as Opportunistic Dual Metric Scheduling Algorithm (ODM) is proposed for LTE Uplink and Downlink. The objective of the algorithm is to maximize the throughput in LTE uplink and to improve the fairness in resource allocation. In order to achieve this, two metrics are used, with the primary metric being throughput centric and the secondary metric being formulated to promote fairness in allocation of resources. Since the user is power limited, a power constraint is added to make the uplink scheduling energy-efficient. For downlink, a new fairness constraint is introduced to improve fairness in resource allocation. The simulation results show that ODM algorithm shows an overall better performance across the performance metrics than BCQI and PF algorithms. Since BCQI scheduler gives the best throughput, the throughput results are compared against BCQI results. It is observed that ODM scheduler achieves more than 91\% throughput values whereas PF algorithm achieves only less than $75 \%$ throughput values for all the simulation scenarios. Upon analysing the schedulers in terms of Jain's fairness index [10], it is observed that PF scheduler shows nearly 88\% and ODM scheduler achieves approximately 70\% which is better than BCQI results of 33\%. Evaluation in terms of transmit power shows ODM uplink scheduler performs better than PF and BCQI schedulers in practical scenarios. Hence, the proposed algorithm is more energy-efficient in uplink and achieves high throughput and fairness values, striking a better trade-off than the other two schedulers for both uplink and downlink.

The rest of the paper has been organized as follows. Section II provides the background and Section III formulates the scheduling problem. Section IV shows the different performance parameters used for evaluating the schedulers. Section V presents the proposed strategy and the proposed algorithms are presented in Section VI. Analysis on the results are presented in Section VII.

\section{Background}

One of the distinguishing features of the LTE system from the previous cellular systems is that LTE is designed to support only Packet Switched (PS) services and has no support for Circuit Switched services. Thus, from a network perspective, the LTE system is purely based on IP architecture, where all the network entities are connected thorough Internet protocol 
(IP). The network can be split into two parts namely, Radio Access Network (RAN) and Core Network (CN). The RAN is consists of Evolved Universal Terrestrial Radio Access Network (EUTRAN) and the CN, namely the Evolved Packet Core Network (EPC). Fig. 1 shows the overall network architecture and the interfaces through which the network elements are connected. To meet the expectation, OFDM, which provides high spectral efficiency and low bit error rate, is chosen as the Radio Access technology. However, OFDM has high Peak to Average Power Ratio (PAPR). Hence, LTE uses OFDMA in downlink and Single Carrier Frequency Division Multiple Access (SCFDMA) transmission in uplink to reduce the power consumption of User Equipments (UE). The building block of LTE is a Resource Block (RB). Each RB is $0.5 \mathrm{~ms}$ in duration and carries 7 OFDM symbols. It is made up of 12 subcarriers occupying a bandwidth of $180 \mathrm{kHz}$ with each subcarrier occupying a bandwidth of $15 \mathrm{kHz}$.

The LTE network architecture (Fig. 1) [4] [11] consists of a radio access network called Evolved UMTS Terrestrial Radio Access Network (E-UTRAN), and a core network called Evolved Packet Core (EPC) Network. E-UTRAN consists of clusters of the evolved NodeB (eNodeB), which connects the UE with the core network through the air interface.

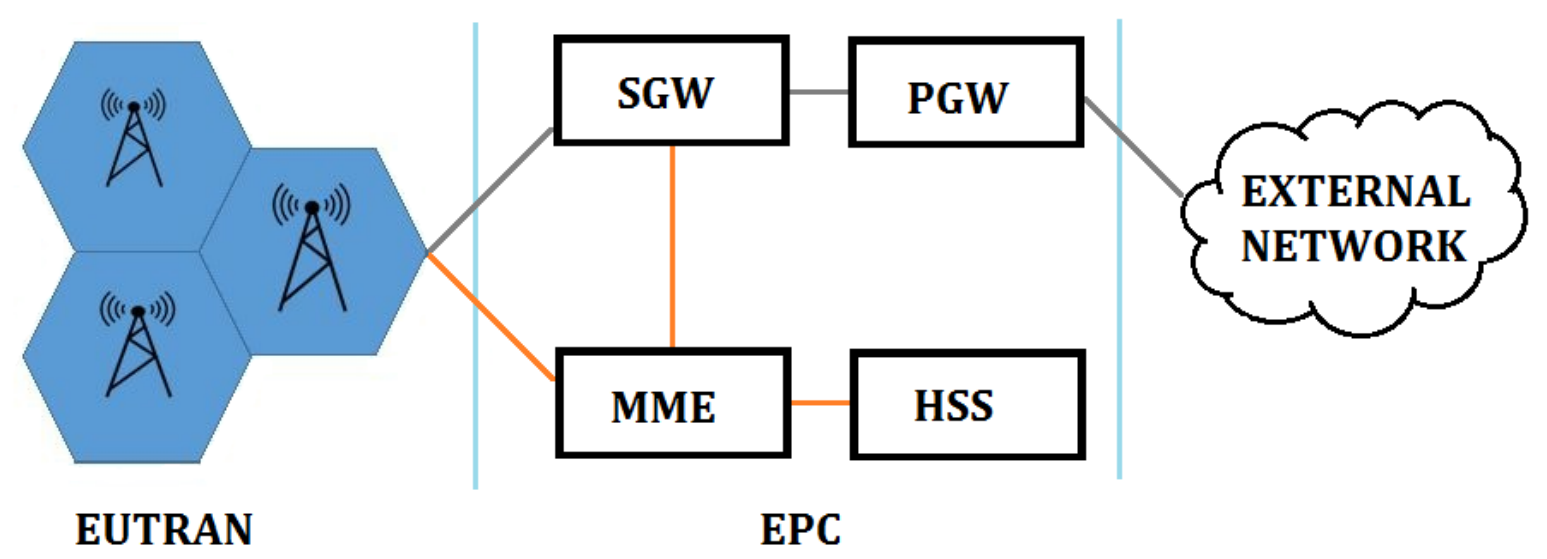

Fig. 1. LTE Network Architecture

The eNodeB is responsible for serving the UE by connecting them with the EPC. The eNodeB is directly connected to the EPC using S1 interface and they are interconnected using $\mathrm{X} 2$ interface. The functionality of Radio Network Controller (RNC), which is present in 2G/3G systems, is decentralized among the eNodeBs. The eNodeB supports the transmission and reception of traffic over the air interface. It is also responsible for the various features such as Radio Resource Control, Mobility Management, and Radio Admission Control [27]. Thus, the dynamic resource allocation, which is the prime focus of this work, is carried out by eNodeB. According to [5], the scheduling algorithms can be classified into two extremes:

- Opportunistic Scheduling focuses on improving the transmission data rate of all the users by exploiting their instantaneous channel conditions.

- Fair Scheduling schemes are designed to promote fairness in allocation by ensuring that every user is allocated with a minimum of radio transmission resources. Hence, the achieved cell throughput will be lesser when compared with the former scheme. 
Most of the algorithms existing in the literature fall in between these two types. The BCQI algorithm is a channel-dependent scheduler which aims to maximize the throughput by allocating more resources for users with high channel quality. It results users with poor channel condition being not allocated with any resources. Thus, it results in an unfair scheduling. PF algorithm assigns priority to users based on their achievable data rate to the average throughput achieved by the user in the previous subframes. Hence, users with poor channel conditions are also allocated with resources.

\subsection{Physical Resource Organization}

The LTE system has the flexibility to choose the bandwidth of operation from $1.4 \mathrm{MHz}$ to $20 \mathrm{MHz}$ depending upon the requirements [5]. The LTE physical resource is defined in both time and frequency domain to provide the flexibility to the resource scheduler. Fig. 2 depicts the LTE physical resource as time-frequency grid. Each subcarrier in LTE has a frequency spacing of $15 \mathrm{KHz}$ [19].

A Physical Resource Block (PRB) has a duration of $0.5 \mathrm{~ms}$ and a bandwidth of $180 \mathrm{KHZ}$ [13]. Thus one PRB 12 consecutive subcarriers in frequency domain and six or seven symbols (depending on the type of cyclic prefix used) in time domain. The PRB is the minimum scheduling size for uplink and downlink resource allocation.

Table I shows the number of LTE resource blocks for various supported system bandwidth according to 3GPP Release 8.

TABLE I. Summary of notations.

\begin{tabular}{|c|c|c|}
\hline System Bandwidth (MHz) & No. of RBs & No. of Subcarriers \\
\hline 1.4 & 6 & 72 \\
\hline 3 & 15 & 180 \\
\hline 5 & 25 & 300 \\
\hline 10 & 50 & 600 \\
\hline 15 & 75 & 900 \\
\hline 20 & 100 & 1200 \\
\hline
\end{tabular}




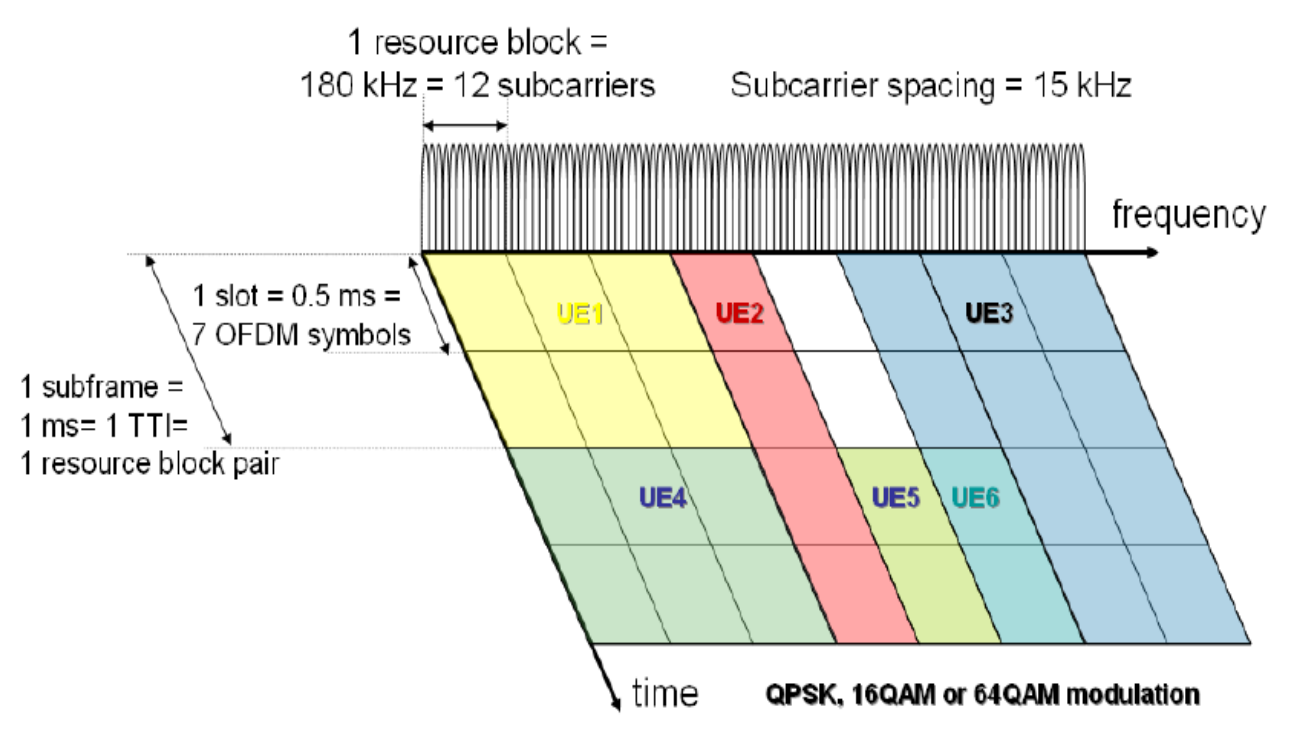

Fig. 2. Physical Resource Representation [11]

A radio frame of LTE [28] has a duration of $10 \mathrm{~ms}$, given that duration of one Transmission Time Interval (TTI) is 1ms. There are two types of LTE radio frames, known as Type 1 and Type 2. The Type 1 radio frames are used for FDD mode and it is divided into 10 subframes where each subframe consists of two slots of duration $0.5 \mathrm{~ms}$ each. The Type 2 radio frames are used for TDD mode and as shown Fig. 3, it is divided into 20 subframes where each subframe has one time slot of duration $0.5 \mathrm{~ms}$.

A Radio Frame = 10ms
\begin{tabular}{|l|l|l|l|l|c|}
\hline$\# 0$ & $\# 1$ & $\# 2$ & $\ldots$. & $\# 18$ & $\# 19$ \\
\hline Slot $=0.5 \mathrm{~ms}$ \\
\hline
\end{tabular}

Fig. 3. LTE Radio Frame 


\section{1) Macrothink}

\section{Problem Definition}

The allocation of resources in LTE can be considered as an optimization problem. The scheduling decision problem can be modelled using a utility function $U$. Let $\bar{n}$ be the number of UEs to be scheduled and $\bar{m}$ denote the RBs available for scheduling. Let $\Delta(n, m)$ denotes that $R B_{m}$ is allocated to $U E_{n}$. The objective is to maximize the utility function $U$. It can be formulated as follows:

$$
\Delta(n, m)=\max _{i \in N}\{U(i, m)\}
$$

Eq. (1) represents that scheduler allocates $R B_{m}$ to $U E_{n}$ if the pair $\left(U E_{n}, R B_{m}\right.$ ) has maximum utility. Each algorithm has specific objectives to fulfill and accordingly the utility function varies [14]. In most of the conventional scheduling algorithm utility function is a resource allocation matrix as shown in Fig. 4, elements of which is formed calculating scheduling metric as per its objective for each (UE,RB) pair.

\begin{tabular}{c|c|c|c|c|}
\multicolumn{1}{c}{$\mathrm{RB}(1)$} & $\mathrm{RB}(2)$ & $\ldots$ & $\mathrm{RB}(\bar{m})$ \\
\cline { 2 - 5 } $\mathrm{UE}(1)$ & $H(1,1)$ & $H(1,2)$ & $\ldots$ & $H(1, \bar{m})$ \\
\cline { 2 - 5 } $\mathrm{UE}(2)$ & $H(2,1)$ & $H(2,2)$ & $\ldots$ & $H(2, \bar{m})$ \\
\cline { 2 - 5 }$\ldots$ & $\ldots$ & $\ldots$ & $\ldots$ & $\ldots$ \\
\cline { 2 - 5 } $\operatorname{UE}(\bar{n})$ & $H(\bar{n}, 1)$ & $H(\bar{n}, 2)$ & $\ldots$ & $H(\bar{n}, \bar{m})$ \\
\hline
\end{tabular}

Fig. 4. Resource allocation matrix

The scheduler receives this matrix as input and for each column $j$, it allocates $j^{\text {th }}$ RB to $i^{\text {th }}$ user if $H(i, j)$ is maximum. The proposed scheduler, ODM consists of two resource allocation matrices which forms the utility function.

The resource allocation process needs to follow a certain constraints for achieving effective system performances. Some of the constraints that are considered in the proposed uplink and downlink scheduling algorithm are explained under the following subsections.

\subsection{Singularity Constraint}

In each subframe, any RB can be allocated to single UE only. RBs cannot be assigned to multiple UEs. However, the converse is possible. Let $\Delta(n, m)$ be the allocation vector of user $n$ to RB $m$ in the given subframe. It can be defined as follows:

$$
\Delta(n, m)=\left\{\begin{array}{l}
1, \text { if } R B_{m} \text { is assigned to } U E_{n} \\
0, \text { if } R B_{m} \text { is not assigned to } U E_{n}
\end{array}\right.
$$


Then, in each subframe, the singularity constraint can be denoted as:

$$
\sum_{i=1}^{N} \Delta(i, m) \leq 1
$$

\subsection{Contiguity Constraint}

SCFDMA has two types of subcarrier mapping. They are Localized FDMA (LFDMA) and Interleaved FDMA (IFDMA). According to [6], in channel-dependent scheduling, LFDMA shows less energy consumption performance than IFDMA when scheduling at the cost of decreased throughput performance. Thus, allocation of contiguous resources will result in increased performance than allocation of intermittent resources. This condition is termed as contiguity constraint. Hence, this constraints is applied to uplink scheduling scheme.

\subsection{Transmit Power Constraint}

According to 3GPP specification [3], the uplink power budgets maximum threshold of any user is equal to $23 \mathrm{dBm}$ for a class $3 \mathrm{UE}$. This can be represented as follows:

$$
\sum_{j=1}^{M} \Delta(n, j) P_{T x}(n) \leq 23 d B m
$$

where $P_{T x}(n)$ is the total transmit power of user $n$ for each subframe. This constraint is applied only to uplink scheduling algorithm.

\subsection{Fairness Constraint}

When compared with the UE, the eNodeB is not power-limited. The continuous resource allocation is not major constraint in LTE downlink. Thus, the algorithm need not satisfy the contiguity constraint.

Hence, a new constraint referred as fairness constraint, is introduced to ensure fairness in resource allocation. The fairness constraint is used in downlink algorithm as a checkpoint before expanding the allocation process. The objective of the fairness constraint is to ensure that in the each TTI, the allocation of additional resources to a user does not result in starvation for other users. This is evaluated by computing the number of resources assigned to the user in the past TTIs. It can be formulated as follows:

$$
\text { Fairness Constraint Index, } \mathrm{F}=1-\frac{\mathrm{RB}_{i}}{\mathrm{~N}_{-} \mathrm{RB}}
$$

where $\mathrm{RB}_{\mathrm{i}}$ is the number of resources allocated to the user $i$ over the past TTI and 
N_RB is the sum of total number of resources available for allocation at each TTI.

Consider a LTE system where the bandwidth is selected to be $1.4 \mathrm{MHz}$. Then, the number of RBs available for allocation, N_RB is equal to 6 . Table II shows the values of F, assuming user allocation over two past TTIs.

TABLE II. Fairness constraint values.

\begin{tabular}{|c|c|}
\hline $\begin{array}{c}\text { Number of RBs assigned over the } \\
\text { past TTI to user } \mathbf{i}, \mathbf{R B}_{\boldsymbol{i}}\end{array}$ & $\begin{array}{c}\text { Fairness Constraint } \\
\text { Index, } \mathbf{F}\end{array}$ \\
\hline 0 & 1.0000 \\
\hline 1 & 0.9167 \\
\hline 2 & 0.8333 \\
\hline 3 & 0.7500 \\
\hline 4 & 0.6667 \\
\hline 5 & 0.5833 \\
\hline 6 & 0.5000 \\
\hline 7 & 0.4167 \\
\hline 8 & 0.3333 \\
\hline 9 & 0.2500 \\
\hline 10 & 0.1667 \\
\hline 11 & 0.0833 \\
\hline 12 & 0.0000 \\
\hline & \\
\hline
\end{tabular}

\section{Evaluation Parameters}

In this section, performance parameters which are used to evaluate the proposed scheduler are examined. Throughput and fairness are the major performance parameters in analyzing any scheduling algorithm. Additionally, a new parameter is introduced in the section to evaluate the scheduler in terms of uplink transmit power.

\subsection{Throughput}

Throughput is the measure of the system's utilization of the resource provided. It is defined as the number of bits effectively transmitted over the air interface from UE to eNB in a given time.

Throughput, $\widehat{T}_{p}$ can be represented as follows:

$$
\widehat{T}_{p}=\frac{I_{b}}{t}
$$

where $I_{b}$ is the number of information bits successfully transmitted from UE to eNB and $t$ is the number of subframes. 


\subsection{Fairness Index}

Jain's fairness is a quantitative measure of fairness in allocation of resources in a system. The idea is proposed by the author [23] to find fairness in resource allocation in shared computer systems. The concept can be extended to LTE system to evaluate the fairness in resource allocation.

Consider a simple LTE system composed of one eNB and $\bar{n}$ users. Let $T_{n}$ be the throughput of the user $n$, where $n$ is subset of $\bar{n}$. Then, Jain's fairness index for resource allocation can be calculated using the following formula:

$$
J=\frac{\left[\sum_{n=1}^{\bar{n}} T_{n}\right]^{2}}{\bar{n} \times\left[\sum_{n=1}^{\bar{n}} T_{n}{ }^{2}\right]}
$$

\subsection{Throughput-to-Power Ratio (TPR)}

One of the key aspect in designing LTE uplink scheduler is consideration for power consumption. UEs have limited battery power and it is vital for scheduling algorithm to allocate the resources in an energy efficient pattern to improve the battery life. According to the specification given in [24], UE Transmit power $\left(P_{T x}\right)$ is modeled as follows:

$$
P_{T x}=\min \left\{P_{\text {max }}, P_{o}+\alpha \cdot P_{L}+10 \log _{10}(M)+\Delta M C S+f(\Delta i)\right\}
$$

The summary of notations used in Eq. (8) is listed in Table III. Due to the limitation in the simulator, a simple model is constructed to evaluate the scheduler in terms of transmit power. Lauridsen et al. [25], have conducted experiments on LTE system to analyze the relation between uplink resource allocation and the UE transmit power. They have concluded that resource allocation directly proportional to the UE transmit power. From [3], the minimum UE transmit power (Pmin) is evaluated as $-30 \mathrm{dBm}$ and maximum UE transmit power (Pmax) is $23 \mathrm{dBm}$. By varying only the number of RBs which are assigned to the users and keeping other parameters fixed, the UE transmit power can be simplified as:

$$
P_{T x}=\min \left\{P_{\text {max }}, P_{\text {min }}+10 \log _{10}(M)\right\}
$$

Throughput-to-Power Ratio (TPR) is defined as the ratio between utilization of resources by each UE to its corresponding transmit power in the given simulation time.

$$
T P R=\left|\frac{T_{n}}{P_{T X}}\right|
$$


TABLE III. Summary of notations.

\begin{tabular}{|c|l|}
\hline Notation & \multicolumn{1}{c|}{ Definition } \\
\hline $\mathrm{P}_{\mathrm{MAX}}$ & The maximum user transmission power \\
\hline $\mathrm{M}$ & The number of allocated PRB at a given TTI \\
\hline $\mathrm{P}_{0}$ & Open loop path-loss power value. \\
\hline $\mathrm{A}$ & Open loop path-loss factor. \\
\hline $\mathrm{P}_{\mathrm{L}}$ & Downlink path-loss measured in the UE \\
\hline$\Delta \mathrm{MCS}$ & Cell dependent factor \\
\hline $\mathrm{f}(\Delta \mathrm{i})$ & User specific closed loop $\mathrm{P}_{\mathrm{MAX}}$ \\
\hline
\end{tabular}

\section{Proposed Strategy}

This section explains the calculation of resource allocation matrices in the proposed ODM algorithm.

\subsection{Calculation of Primary and Secondary Matrices}

As explained in Section II, the utility function of any scheduling algorithm gives priority to specific parameters. For example, Round Robin scheduler follows fairness as primary metric by allocating the same number of RBs for all the users. Thus, the nature of the algorithm is determined by the utility function. The objective of the proposed algorithm is to improve the system performance in terms of throughput at the same time to provide fair allocation of resources.

ODM scheduler consists of two resource allocation matrices, primary metric matrix $\left(H_{1}\right)$ and secondary resource allocation matrix $\left(\mathrm{H}_{2}\right)$. The metric is the improvised version of Opportunistic Proportional Fairness metric proposed in [26] for any wireless communication. Consider an LTE system comprising of single eNodeB and $\bar{n}$ UEs. Let the available bandwidth consists of $\bar{m}$ RBs. In the given subframe $t$, let $R_{n}(t)$ be the bit rate of the user $n$ and $T_{n}$ be the average throughput of user $n$ over previous subframes.

The element $H(n, m)$ in the $\bar{n} \times \bar{m}$ resource allocation matrix $(H)$ is the scheduling metric, calculated for the (UE, RB) pair $U E_{n}$ and $R B_{m}$ by the following equation:

$$
H(n, m)=\left\{\alpha \cdot\left(\frac{R_{n}(t)}{\dot{T}_{n}}\right)+\beta \cdot\left(\frac{R_{n}(t)}{\max _{\forall i}\left(R_{i}(t)\right)}\right)\right\}
$$

The factor $\frac{\mathrm{R}_{\mathrm{n}}(\mathrm{m})}{T_{n}}$ represents the PF scheduling metric and the factor $\frac{\mathrm{R}_{\mathrm{n}}(\mathrm{m})}{\max _{\forall \mathrm{i}}\left(\mathrm{R}_{\mathrm{i}}(\mathrm{m})\right)}$ represents the BCQI scheduling metric. 


\section{Macrothink}

\subsection{Evaluation of Metric Coefficients}

The effectiveness of the scheduling metric depends on the value of $\alpha$ and $\beta$. When $\alpha=1$ and $\beta=0$, then metric becomes PF scheduler. Similarly, when $\alpha=0$ and $\beta=1$, the metric becomes BCQI scheduler. However, the author in [26] has fell short in explaining the reason behind assuming the values of $\alpha$ and $\beta$ to be 1.5 and 1 . In order to overcome this assumption, experiments were performed to determine the values of the primary and secondary coefficients for uplink and downlink respectively.

The simulation scenario is setup with one eNodeB and 20 randomly distributed UEs experiencing different average SNRs. The performance of the algorithm is analyzed in terms of throughput and fairness varying both $\alpha$ and $\beta$ in the range of 0.25 to 1 in steps of 0.25 . Fig. 5 and 6 depicts the throughput and fairness performance result of LTE uplink averaged over 20 simulation iterations respectively.

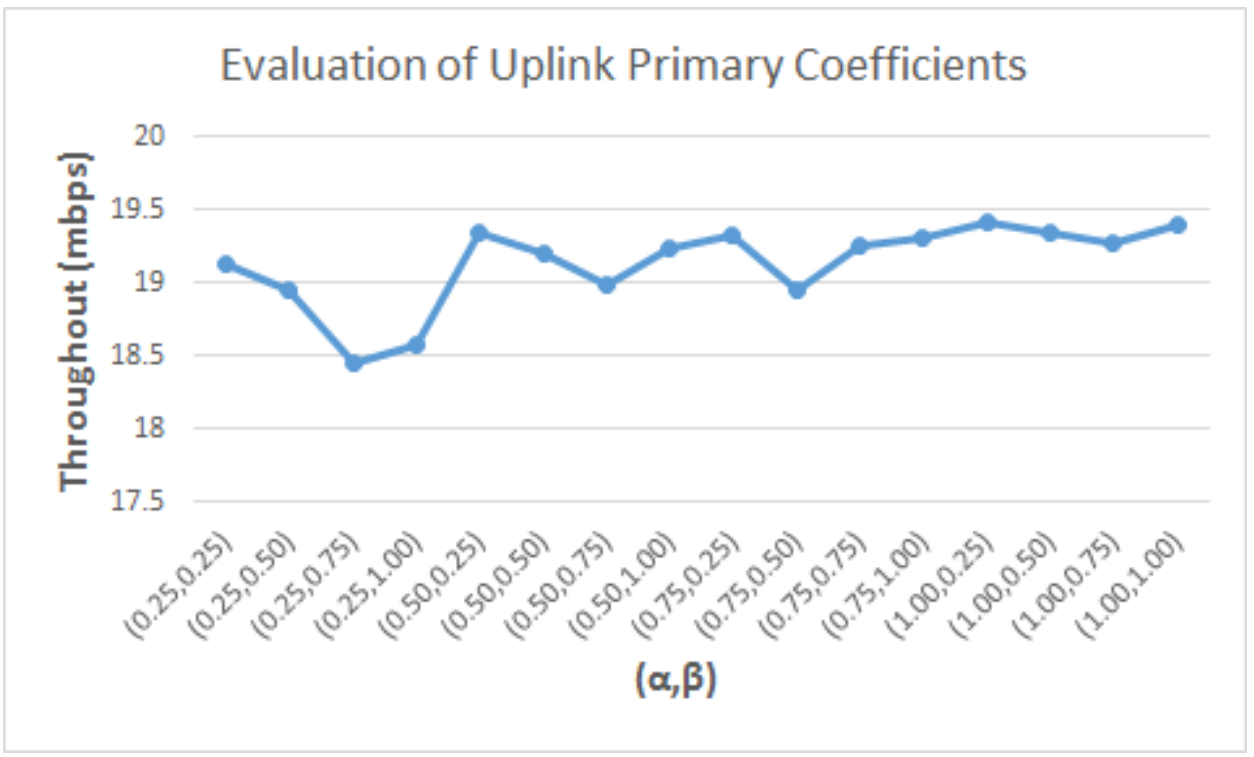

Fig. 5. Evaluation of uplink primary coefficients

From the results, the peak values are chosen to be the coefficients of metric matrices $H_{1}$ and $\mathrm{H}_{2}$. The primary metric is throughput centric. Hence, from Fig. 5 the coefficients pair which provides the peak value for throughput are chosen. Similarly, the secondary metric is chosen to be fairness centric. Hence, the coefficients pair which provides maximum fairness index in Fig. 6 are chosen. Therefore, from the results the coefficients of uplink primary and secondary metrics are chosen to be $(1,0.25)$ and $(0.75,0.5)$ respectively. 


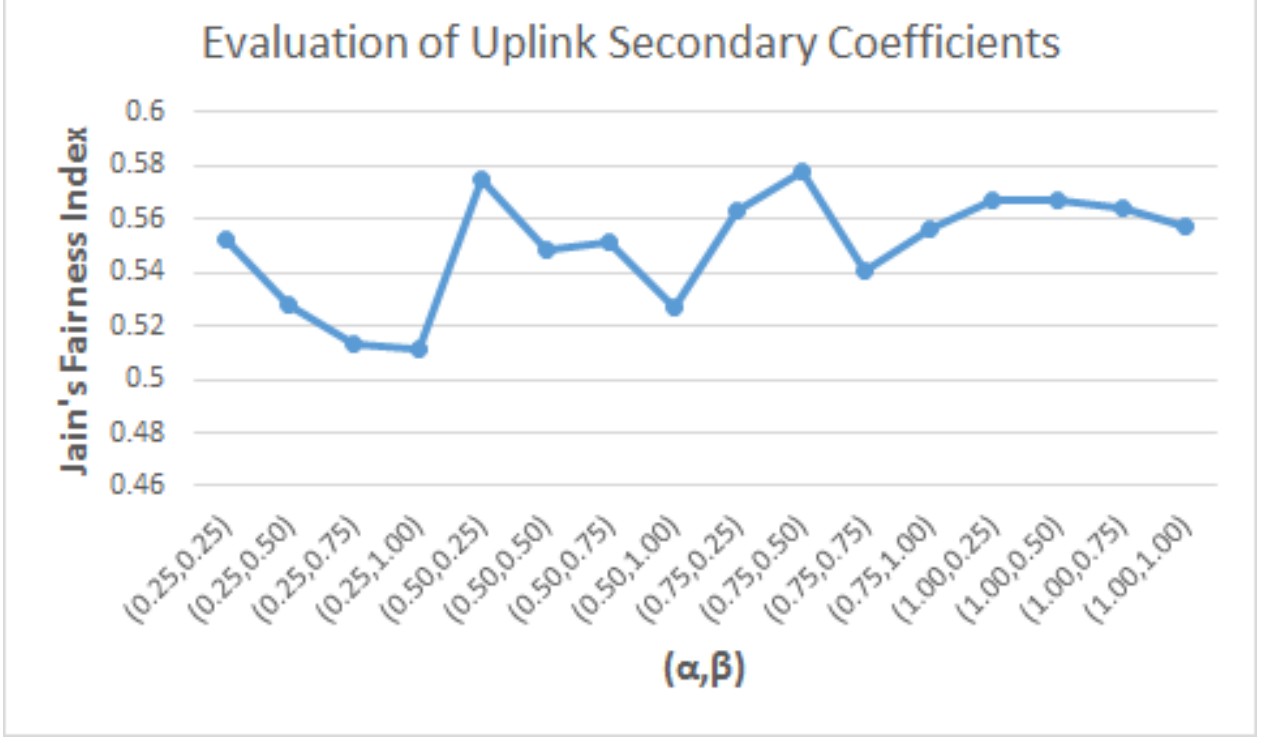

Fig. 6. Evaluation of uplink secondary coefficients

The experiment is repeated for downlink scenario with the same simulation model, consisting of one eNodeB and 20 UEs. The UEs are randomly distributed and they experience average SNRs different from one another. The throughput and fairness performance of the system is evaluated for the decision metric given by Eq. (11) by varying both $\alpha$ and $\beta$ in the range of 0.25 to 1 in steps of 0.25 . The throughput and fairness results are depicted in Fig. 7 and Fig. 8. Similar to the uplink scenario, the coefficients of $H_{1}$ and $H_{2}$ matrices are chosen from the results to be $(1,0.75)$ and $(1,0.25)$ respectively.

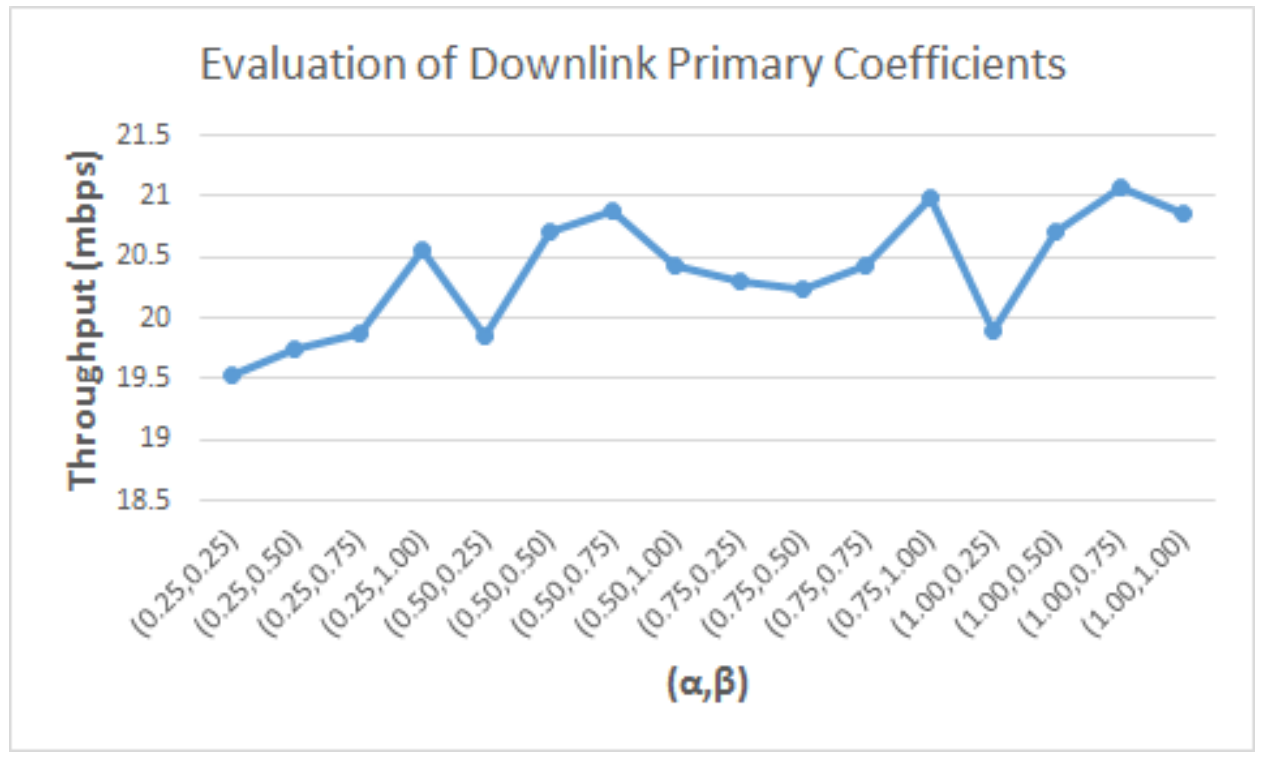

Fig. 7. Evaluation of downlink primary coefficients 


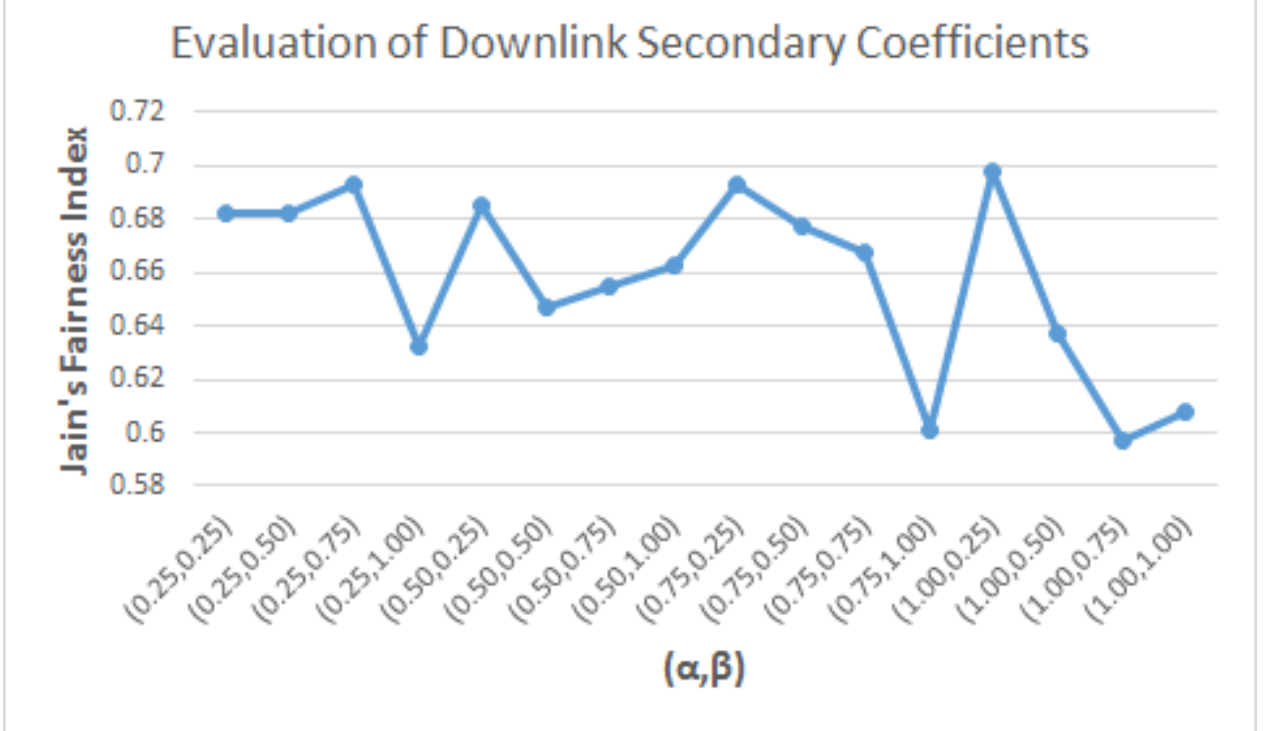

Fig. 8. Evaluation of downlink secondary coefficients

\section{ODM Uplink and Downlink Algorithm}

The channel-aware scheduling exploits the knowledge of the channel conditions to allocate resources such that the system throughput can be maximized. The achievable data rate of the user ' $n$ ' over the RB ' $m$ ' can be computed using Shannon's Channel Capacity, provided the Signal-to-Noise Ratio (SNR) between the user ' $n$ ' and the RB ' $m$ ' is known. It is represented by the formula below:

$$
\mathrm{R}_{\mathrm{n}}(\mathrm{m})=\log (1+\mathrm{SNR}(\mathrm{n}, \mathrm{m}))
$$

This section presents the design and implementation of a channel aware uplink and downlink algorithm which aims to provide a good trade-off between throughput and fairness in allocation.

\subsection{ODM downlink scheduling algorithm}

The algorithm proposed in the previous section is specific to uplink scheduling. In order to utilize the algorithm for LTE downlink, a few modifications are required. For example, the contiguous allocation of resource is not a constraint for downlink. Hence, an alternate rule is used for expanding the resource allocation for the users.

A pattern-based scheduling strategy, search-tree pattern is used in the proposed downlink algorithm. The algorithm proposed in this section introduces a new method for constructing a search-tree pattern. The search-tree used is a simple pattern with one root node and two branch nodes. Hence, the complexity is very less when compared with the pattern constructed in [21]. This section explains the modification done to ODM scheduling discipline for effective scheduling for LTE downlink. 
The modified ODM algorithm for downlink consists of two levels. The first level uses the primary allocation matrix, $H_{1}$, to find the UE-RB pair which has the maximum metric value. In the second level, a search-tree is built using both primary and secondary allocation matrices, $\mathrm{H}_{1}$ and $\mathrm{H}_{2}$, to expand the allocation of resources to user from the first level. The search-tree pattern used in the algorithm accepts the user index from the first level of the algorithm as input. This forms the root node of the search-tree. A root node has two branch nodes. Each branch node in the pattern has a node score. The node score of the left branch node is calculated using the matrix $H_{1}$ and the node score of the right branch node is calculated using the matrix $\mathrm{H}_{2}$. Node score can be defined as the ratio between metric value of the RB with maximum value for the selected UE (row-wise maximum) and the maximum metric value that $\mathrm{RB}$ (column-wise maximum). If $R B_{m}$ is the best $\mathrm{RB}$ for the user $U E_{n}$, then the node score of the node formed by user UEn and RBm can be formulated as follows:

$$
\Omega s(\mathrm{k})=\frac{H_{\mathrm{k}}(\mathrm{n}, \mathrm{m})}{\max _{\forall \mathrm{i}} \mathrm{H}_{\mathrm{k}}(\mathrm{i}, \mathrm{m})}
$$

where the value of $\mathrm{k}$ varies from 1 and 2 . The root node in the search-tree forms the branch nodes, provided the fairness constraint is satisfied.

Consider the following $4 \times 5$ primary and secondary allocation matrices, $H_{1}$ and $H_{2}$, where each row represents the UE and each column represents the RB as shown in Fig. 9.

$$
H_{1}=\left[\begin{array}{lllll}
3.5 & 7.7 & 5.1 & 0 & 1.3 \\
0.5 & 1.2 & 2.8 & 0 & 1.5 \\
1.7 & 4.3 & 5.4 & 0 & 2.4
\end{array}\right] \quad H_{2}=\left[\begin{array}{lllll}
2.7 & 6.9 & 4.3 & 0 & 0.3 \\
4.4 & 3.1 & 3.8 & 0 & 3.5 \\
0.9 & 9.5 & 7.6 & 0 & 2.7
\end{array}\right]
$$

Fig. 9. Example $H_{1}$ and $H_{2}$ Matrices for Search-Tree Construction

Assume that the UE index 2 is assigned with RB index 4 in the first level of the algorithm. Hence, column 4 is made zero in both $H_{1}$ and $H_{2}$. The primary root node obtained from first level of the algorithm is Node[2,4]. Considering the fairness constraint is satisfied, the left branch node's score can be calculated using $H_{1}$ matrix as follows: 


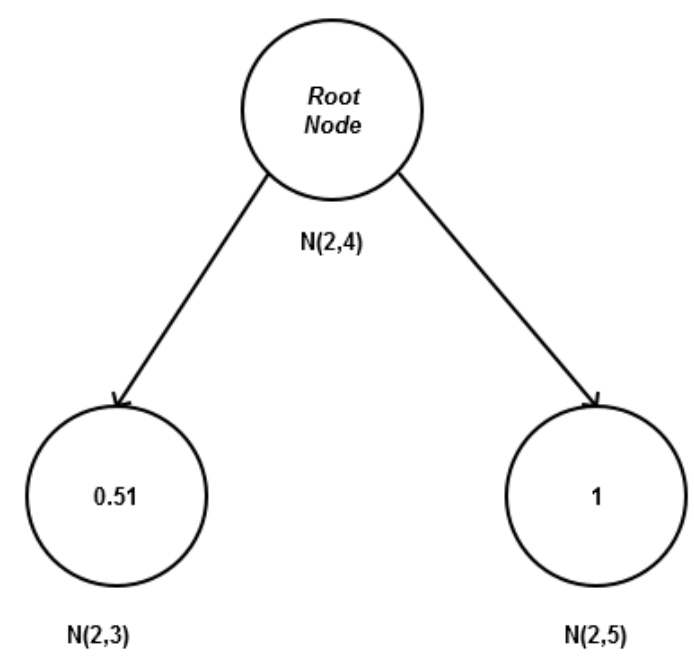

Fig. 10. Sample Search-Tree Pattern

The RB index with maximum value for the UE index 2 in $H_{1}$ is $3(\mathrm{~N}[2,3]=2.8)$. The maximum metric value for RB index 3 falls on UE index $3(\mathrm{~N}[3,3]=5.4)$. Hence, the left branch node's score is

$$
\Omega \mathrm{S}=\frac{2.8}{5.4}=0.51
$$

Similarly, the right branch node's score is calculated using Eq. (13) from $H_{2}$ matrix. The $\mathrm{RB}$ index with maximum value for the UE index 2 in $H_{2}$ is $1(\mathrm{~N}[2,1]=4.4)$. The maximum metric value for RB index 1 also falls on UE index 2 . Hence, the left branch node's score is

$$
\Omega \mathrm{S}=\frac{4.4}{4.4}=1
$$

Hence the left branch node's score is 0.51 and the right branch's score is 1 . Fig. 10 shows the branch nodes constructed for Node[2,4] in the scenario mentioned above.

The pseudo-code explaining the proposed ODM scheduling algorithm for LTE downlink. The algorithm accepts the metric coefficients $\left(\alpha_{1}, \beta_{1}\right)$ and $\left(\alpha_{2}, \beta_{2}\right)$ as inputs. Using these coefficients values, the primary and secondary allocation matrices are computed. The first level of allocation is the initialization stage. The algorithm collects the input that are required to process resource allocation. In this level, the primary and the secondary resource allocation matrices get instantiated. The second level is the core scheduling logic, where the primary and secondary allocation matrices are employed to effectively allocate the resource. Both $H_{1}$ and $H_{2}$ are used build the search-tree. Fig. 11 shows the flowchart of ODM downlink scheduling algorithm. 


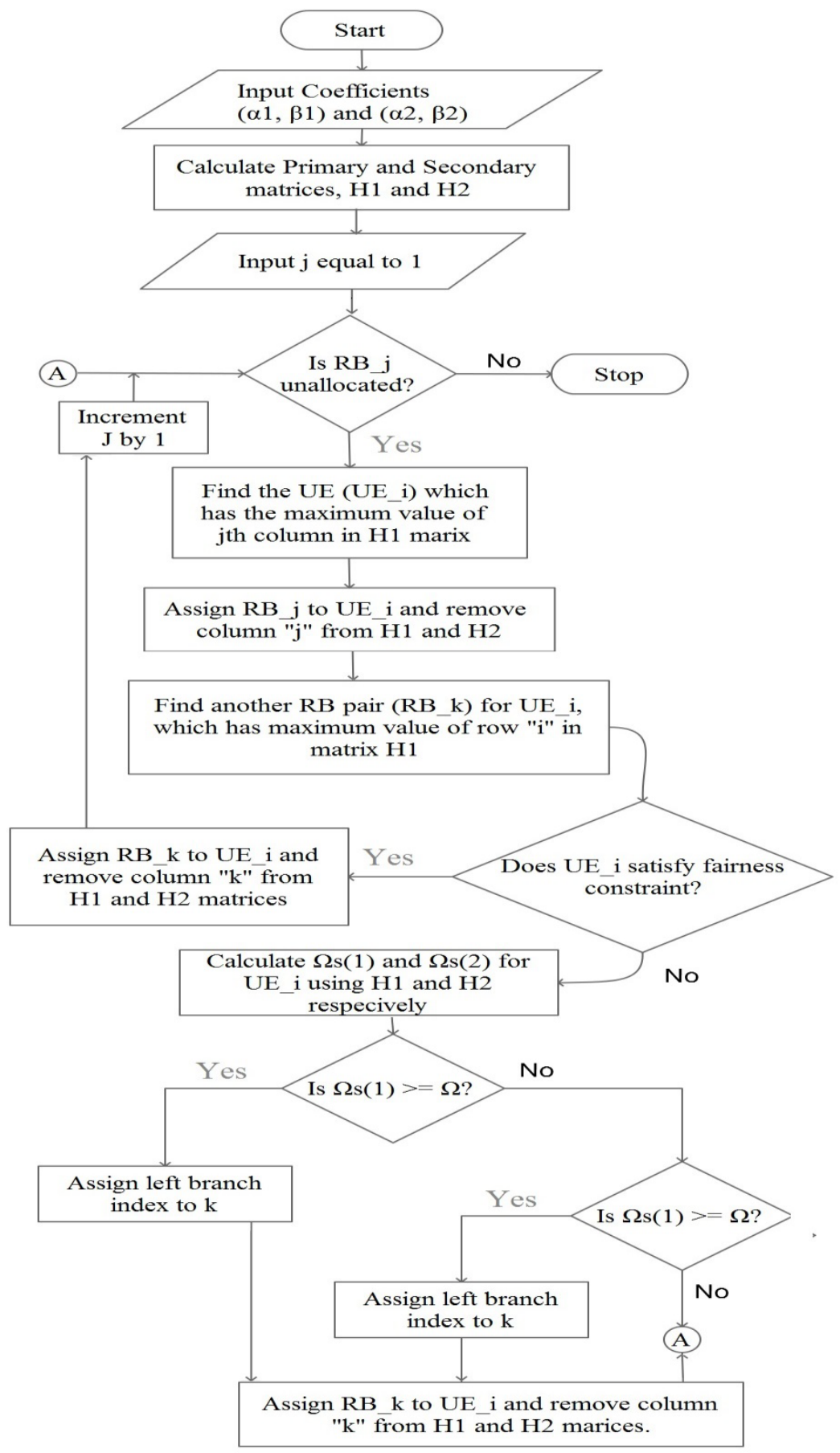

Fig. 11. ODM Downlink Algorithm

One of the important factor in the second level of the algorithm is node score cut-off, $\Omega$ and fairness constraint index, $F$. The values of $F$ and $\Omega$ are always less than or equal to one and their value affect the system performance. In addition to singularity constraint, the effectiveness of the algorithm is increased by considering the proposed fairness constraint.

\subsection{ODM uplink scheduling algorithm}

Similar to the downlink algorithm, the uplink scheme consists of two resource allocation 


\section{Macrothink}

matrices, $H_{1}$ and $H_{2}$. The primary and secondary matrices are employed to provide an efficient distribution of resources among the active users, under the constraints mentioned under Section III. Both the primary and secondary matrices are used to allocate the best resources following the contiguity constraint. The flowchart of the proposed uplink scheduling algorithm is shown in Fig. 12.

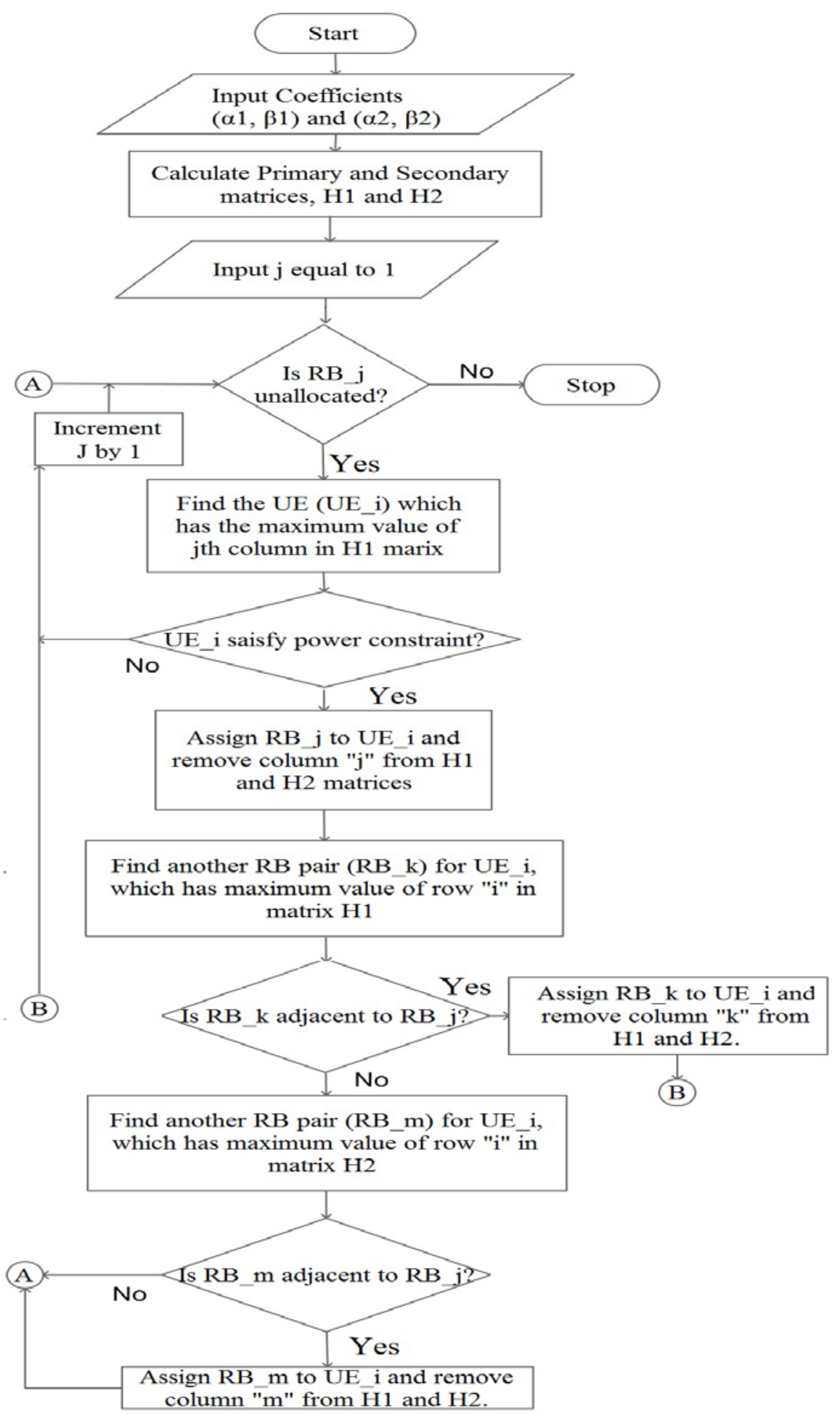

Fig. 12. ODM Uplink Algorithm 


\section{Macrothink}

\section{Results and Discussion}

This section presents the simulation results consisting of performance of ODM uplink and downlink algorithm is compared against standard scheduling schemes, namely, BCQI and PF algorithm. The simulation is executed using MATLAB-based [10] LTE simulator known as "Vienna LTE Link Level Simulator" [8] [9]. The comparison between the scheduling schemes is done based on the parameters mentioned in Section IV.

\subsection{Simulation Model}

The simulation setup consists of a single cell LTE network with one eNB and the network is evaluated for different scenarios varying the number of UEs in the cell. Since the simulator does not have a GUI, Fig. 13 is shown for the depiction of the simulation model with single LTE cell and randomly distributed UEs. All the UEs in the cell are modelled to have average SNRs ranging from 30 to $0 \mathrm{~dB}$ and they are randomly deployed in the cell. To analyze the algorithm in better way the SNRs are assigned to users in decreasing order from UE index 1 to the last UE. Also, to simplify the simulation, it is assumed that all the users have infinitely backlogged data to transmit. The simulation parameters used in analyzing the performance of the algorithm are tabulated in Table VI.

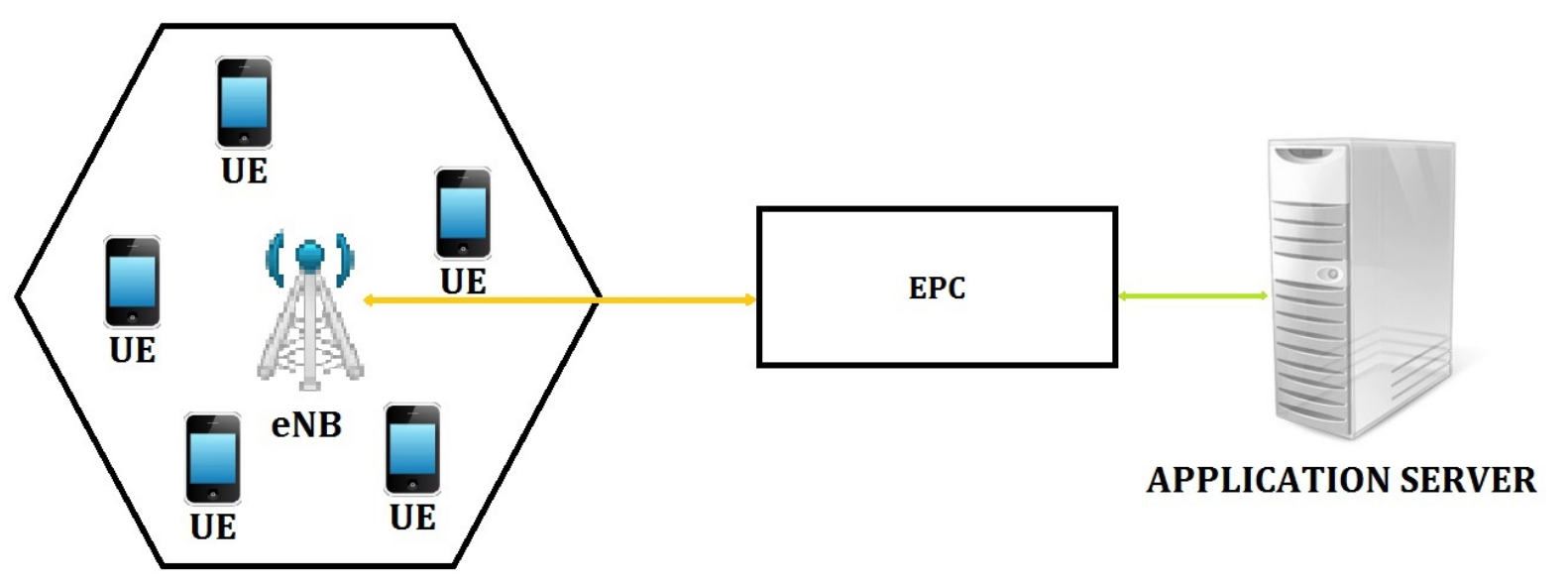

Fig. 13. Simulation setup

The simulation setup considers five simulation scenarios are modelled by varying the number of UEs (10, 15, 20, 25 and 30 respectively) in the cell. The different simulation scenarios are chosen with the intention to analyze the performance of the algorithm when the number of active users less than, equal to and greater than the number of available resources. Each scenario is simulated over 20 iterations and the results are averaged. From the results, it is observed that the network load does not affect the performance of ODM scheduler. The results of all three scenarios follow similar pattern as expected. 
TABLE VI. Simulation Parameters.

\begin{tabular}{|l|l|}
\hline \multicolumn{1}{|c|}{ Parameter } & \multicolumn{1}{c|}{ Value } \\
\hline System bandwidth & $3 \mathrm{MHz}$ \\
\hline Number of RBs & 15 \\
\hline Number of users & $10,15,20,25,30$ \\
\hline TTI Duration & $1 \mathrm{~ms}$ \\
\hline Average SNR of users 1 to 20 & $30 \mathrm{~dB}$ to 0 dB \\
\hline Schedulers & BCQI, ODM, PF \\
\hline
\end{tabular}

\subsection{Throughput Analysis}

The throughput analysis of the proposed and standard scheduling algorithm is discussed in this section.

\subsubsection{LTE Uplink Throughput Analysis}

Fig. 14 shows performance of BCQI, ODM and PF scheduler in terms of throughput calculated using Eq. (6). BCQI scheduler sets the bench mark for the maximum achievable throughput as its only objective is to maximize the throughput. Consider the first simulation scenario which has 10 UEs in the cell. On comparing with BCQI scheduler, it is observed that ODM scheduler achieves throughput value approximately 94\% while PF algorithm achieves throughput values of nearly $77 \%$. This gain in throughput when compared with PF scheduler is due to the $\beta$ factor in the metric. This factor assigns extra priority to users with better channel condition and hence, the increased throughput. Also, from Fig. 14, it can be observed that, the throughput performance follows the same pattern irrespective of the number of UEs in the cell. ODM scheduler achieves nearly 92\% throughput on an average for all the simulation scenarios while $\mathrm{PF}$ achieves an average around $75 \%$ throughput when compared with BCQI scheduler.

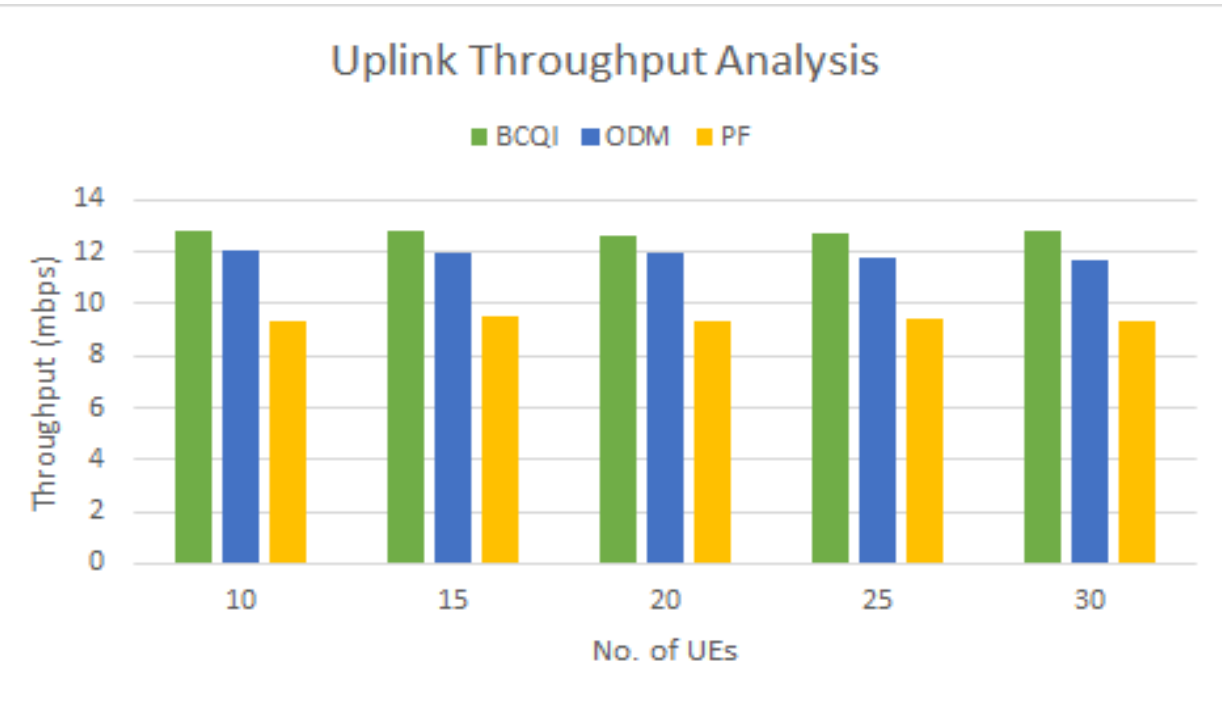

Fig. 14. Throughput Analysis of Uplink Scheduling Algorithm 


\subsubsection{LTE Downlink Throughput Analysis}

The effect on throughput performance of the proposed algorithm for the node score cut-off, $\Omega$, ranging from 0.25 to 1.0 is analyzed in Fig. 15 . The value of $\Omega$ decides the effectiveness of the algorithm in finding the best UE-RB pair. When $\Omega=1$, it signifies that the RB selected has the maximum metric value in the resource allocation matrix and it is expected to provide the best performance for a given UE.

When $\Omega<1$, it signifies that the selected RB is the best for the given UE, however, the selected RB is expected to deliver better performance with another UE. The impact of value of $\Omega$, assuming $\mathrm{F}$ being set to 1 , can be observed from the results shown in Fig. 15. The throughput performance of the algorithm reduces with lower values of $\Omega$. As explained, this is because when node score cut-off is less than 1 , the RB is mapped to an UE although the selected RB will give better performance with another UE.

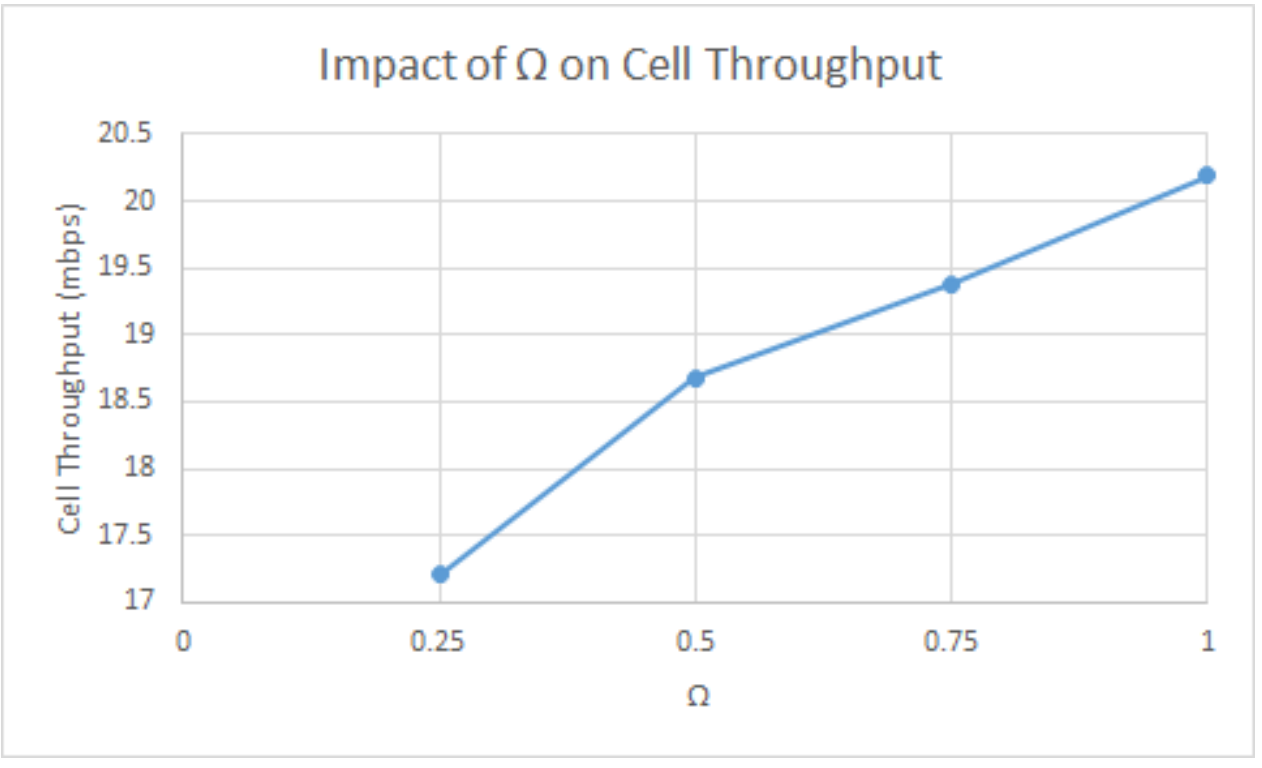

Fig. 15. Impact of $\Omega$ on Cell Throughput

The node score cut-off determines the effectiveness of the proposed algorithm in terms of throughput. The throughput performance shown in Fig. 16 of the proposed ODM downlink algorithm is similar to performance discussed for uplink scenario. The performance of proposed algorithm falls marginally short of BCQI algorithm, however, the results are better than PF algorithm. Assuming that BCQI algorithm gives maximum result, tor the simulation scenario with 10 UEs with SNR averaged between $30 \mathrm{~dB}$ and $0 \mathrm{~dB}$, the proposed algorithm provides around 93\% throughput assuming BCQI to give the maximum performance, whereas PF algorithm nearly $76 \%$ throughput results. The results are quite similar for simulation scenarios with 15, 20, 25 and 30 UEs in the cell. 


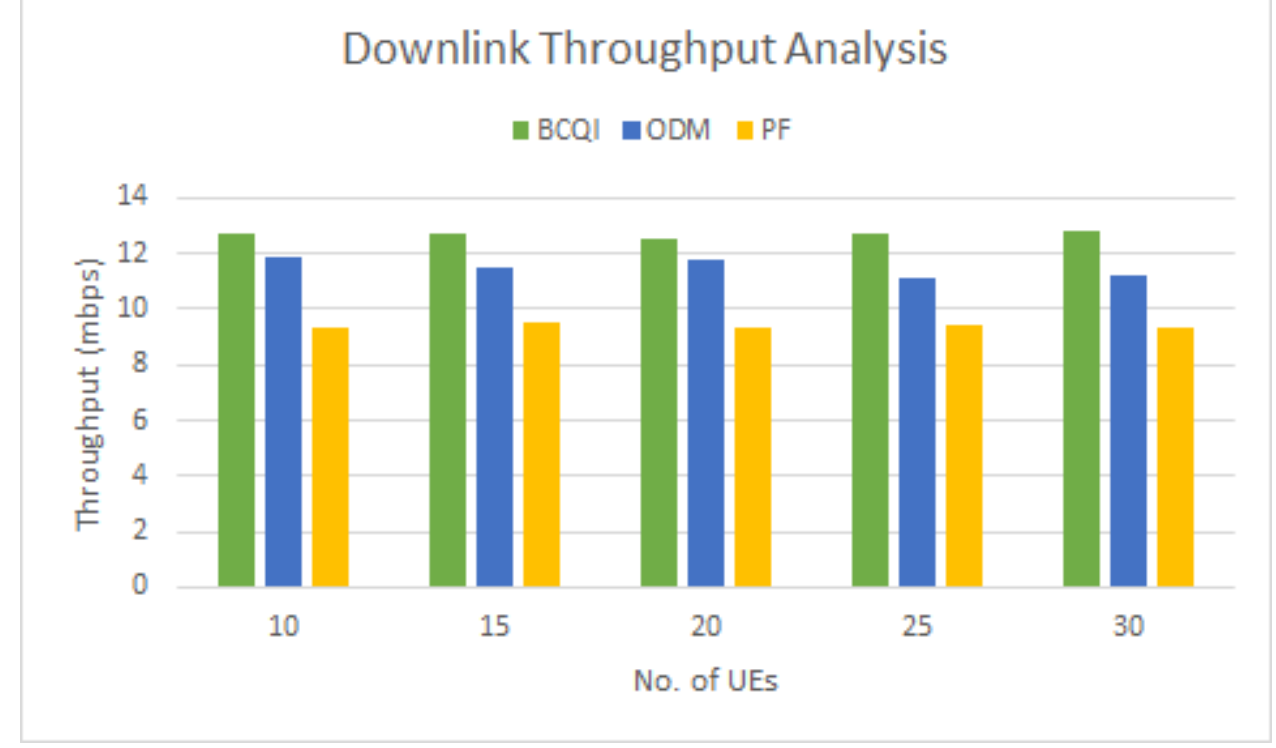

Fig. 16. Throughput Analysis of Downlink Scheduling Algorithm

\subsection{Fairness Analysis}

The performance of the schedulers in terms of fairness is evaluated using the Eq. (7). The fairness results are explained in this section.

\subsubsection{LTE Uplink Fairness Analysis}

Fig. 17 shows the fairness analysis done using Jain's fairness metric. The range of Jain's fairness index is between $1 / N \leq J \leq 1$ where $\mathrm{N}$ is the number of UEs in the cell. As expected, BCQI shows poor fairness due to starvation of users with bad channel condition.

\section{Uplink Fairness Analysis

$$
\text { —CQI } \square O D M \square P F
$$

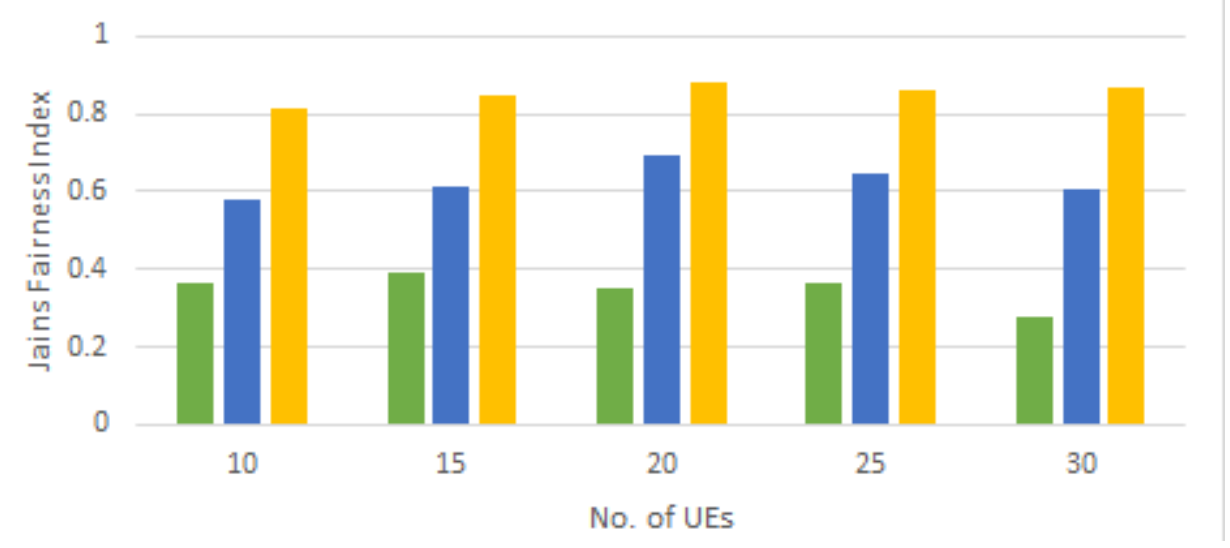

Fig. 17. Fairness Analysis of Uplink Scheduling Algorithm

Comparing with BCQI results, ODM scheduler shows nearly 33\% to 35\% increase in fairness index value (more than 0.7 in all three scenarios), however, shows mildly less 


\section{Al Macrothink}

fairness value than PF scheduler. This is a trade-off as users with better channel conditions are given more preference to achieve better throughput performance. Similar to Fig. 14, the fairness performance of all three schedulers are also is similar for all three simulation scenarios, however, it mildly decreases with the increase in number of UEs in the cell. This behavior is expected as the network volume increases, the demand for resource increase thereby more resources are given to users with better channel condition.

\subsubsection{LTE Downlink Fairness Analysis}

Fig. 18 depicts the fairness index results of the proposed algorithm for values of $F$ ranging from 0.25 to 0.90 , with $\Omega$ being set to 1.0. As the $F$ value decreases, the resource allocation process is extended for users. Thus, the Jain's fairness index value of the system decreases with the decrease of fairness constraint index $(\mathrm{F})$ value in the proposed algorithm. From the results discussed, it can be concluded that for the effective performance of the downlink algorithm, the values of $(\mathrm{F}, \Omega)$ must be equal to $(0.90,1.0)$.

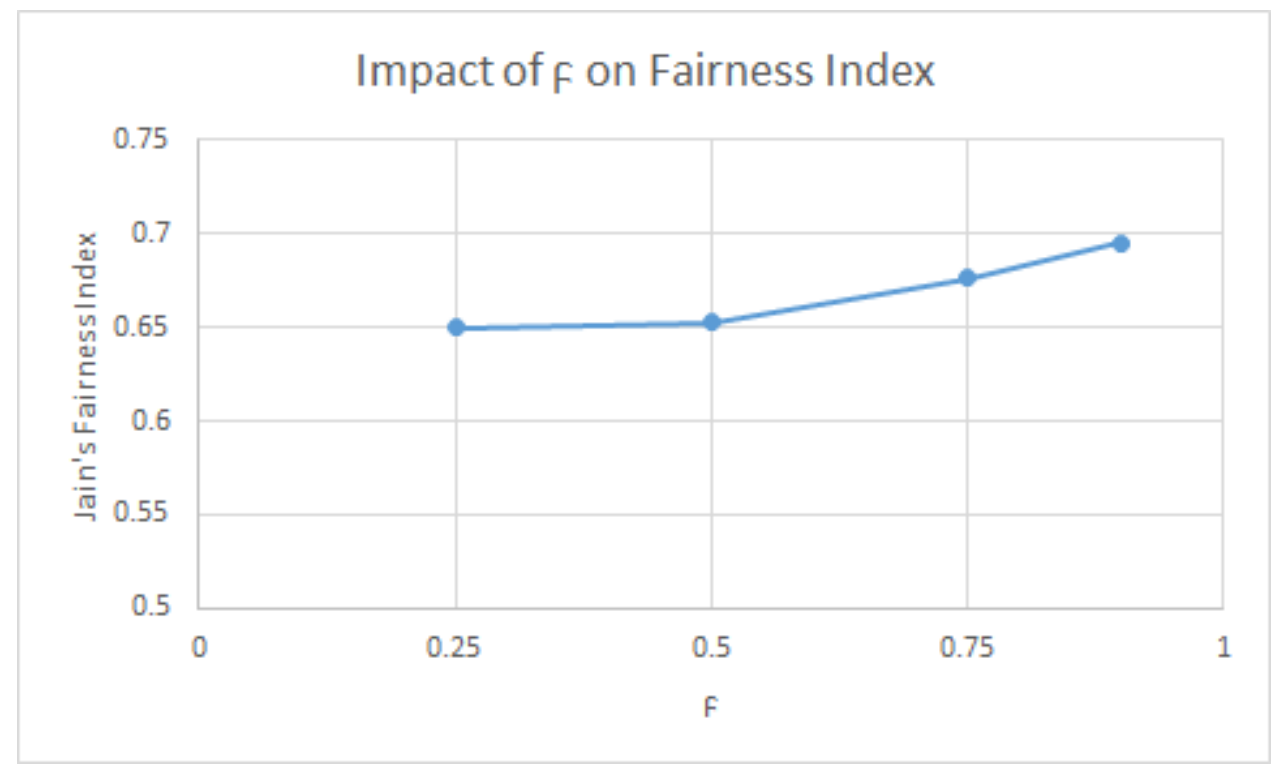

Fig. 18. Impact of $F$ on Fairness Index

The performance of the proposed algorithm in terms of fairness is dependent on the value of F. Although the performance of ODM algorithm falls short of PF algorithm, the fairness index value is approximately $36 \%$ to $45 \%$ more than BCQI algorithm. The fairness performance of the algorithm improves as the number of users are increased in the cell and the fairness index value of the proposed algorithm reaches nearly 0.7 and comparable with PF algorithm value of 0.8 .

Fig. 19 shows the fairness index value of the proposed algorithm and standard downlink algorithms for simulation scenarios with 10, 15, 20, 25 and 30 UEs. 


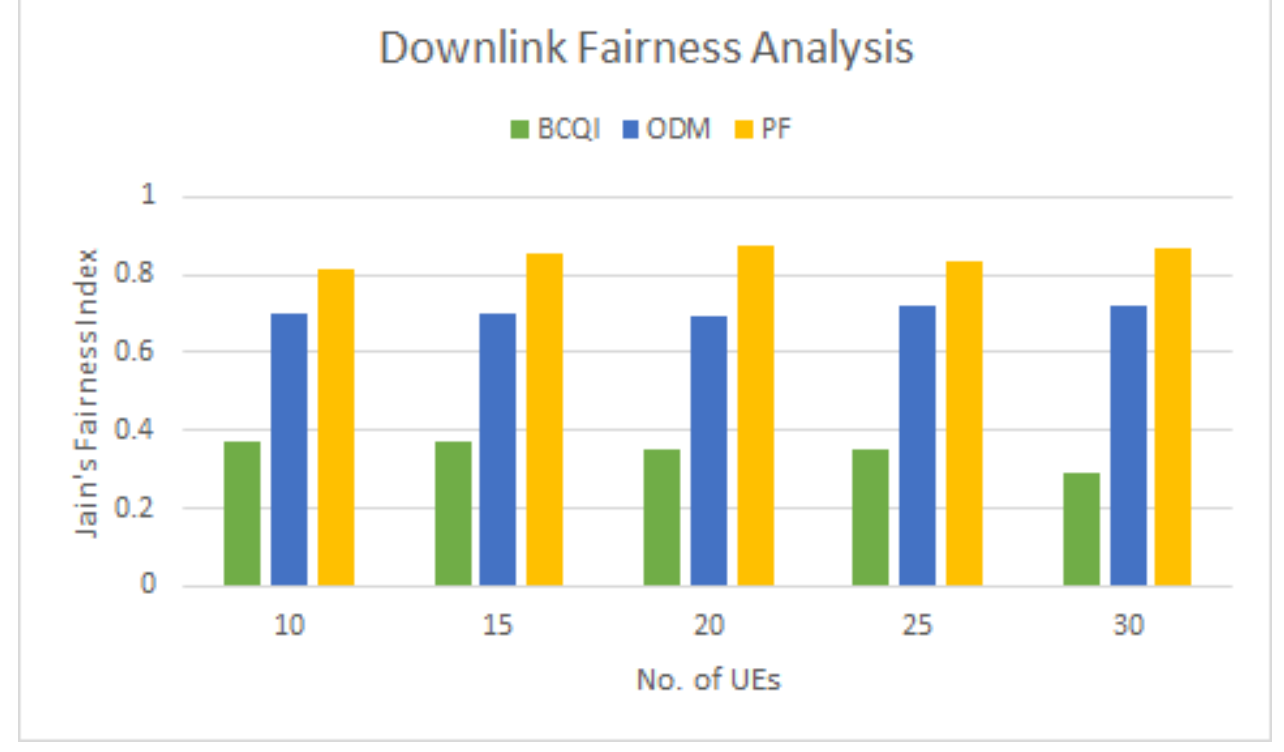

Fig. 19. Fairness Analysis of Downlink Scheduling Algorithms

\subsection{Throughput-to-Power Ratio Analysis}

The Throughput-to-Power Ratio of each user is evaluated using Eq. (10). The performance of all three schedulers in 20 UEs per cell scenario is shown in Fig. 14 and Fig. 15. The results of TPR values in similar for all the simulation scenarios (not provided to avoid repetition). In order to study the performance better, the results are divided into two cases.

Case 1: Users with best channel condition

The TPR results of all three schedulers for users with best channel conditions is shown in Fig. 20. From the figure, it can be observed that users with best channel condition show maximum TPR value. BCQI scheduler is the best performer in this case while ODM scheduler shows marginally better performance than PF scheduler. This is observed because BCQI favors users with good channel conditions. At poor channel conditions, all three schedulers show fall in TPR values. 


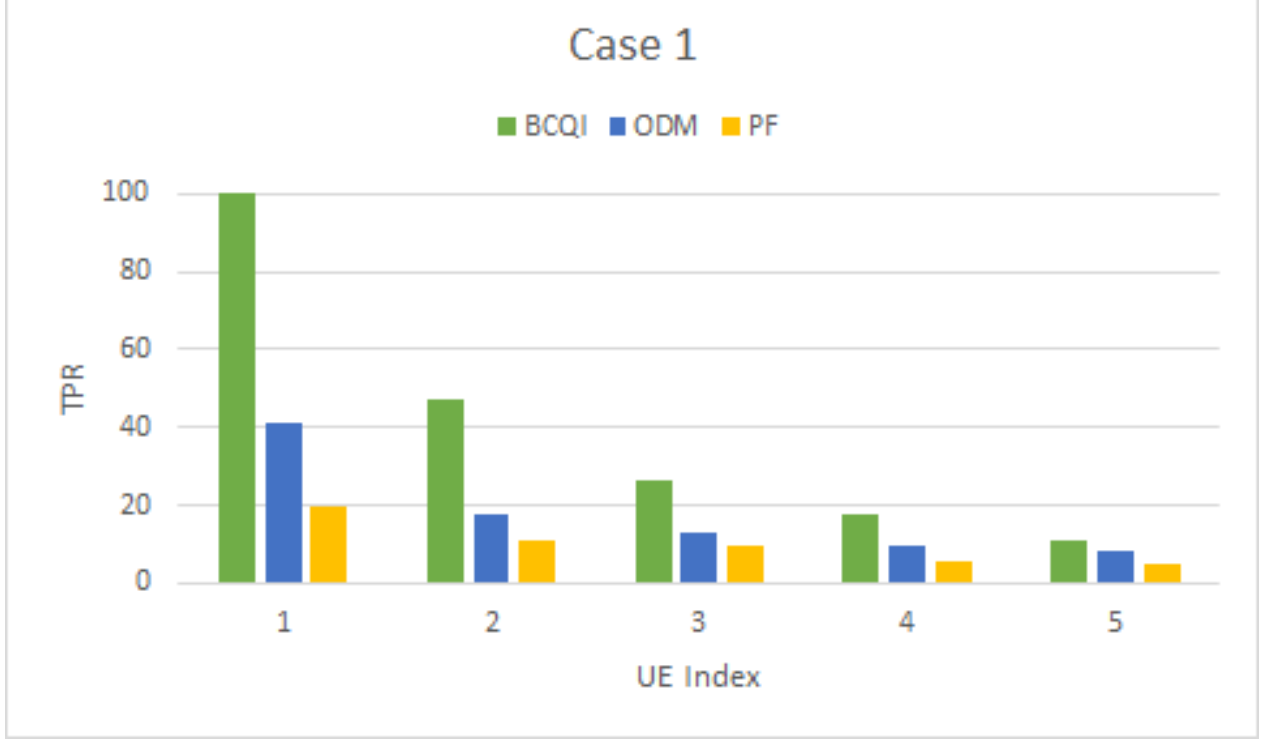

Fig. 20. Throughput-to-Power Ratio Analysis

(Users with best channel condition)

Case 2: Users with good/average/bad channel condition

Fig. 21 shows the Case 2 TPR results of all three schedulers. ODM algorithm shows much improved performance for users under Case 2. The results indicate that the proposed algorithm outperforms other two schedulers for users with good/ average channel conditions and marginally falls short for users with poor channel condition when compared with PF scheduler.

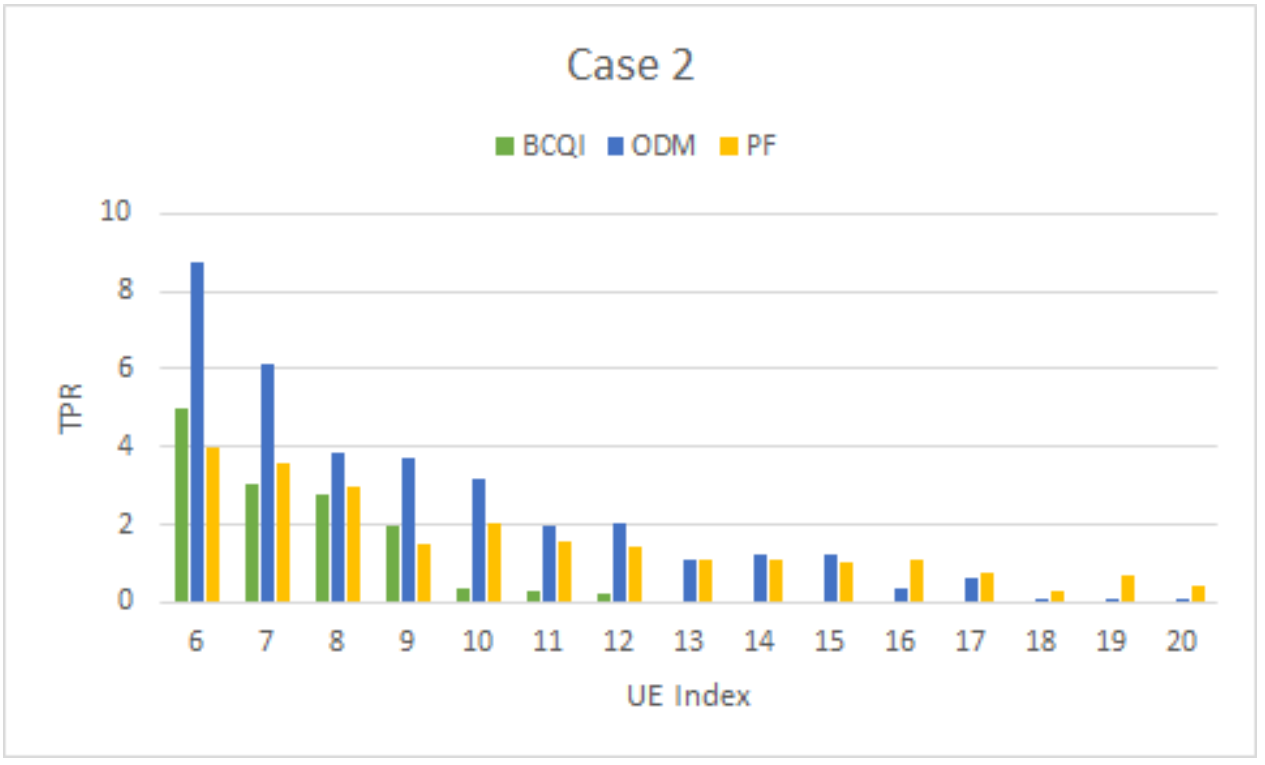

Fig. 21. Throughput-to-Power Ratio Analysis

(Users with good/average/bad channel condition) 
Another important point to be noted is the drastic fall in TPR values of BCQI scheduler. In stark contrast to the performance in Case 1, BCQI scheduler shows almost null value for users in Case 2. As explained earlier, this behavior is due to the unfair resource mapping favoring users with best channel condition. Thus, comparing both the cases, ODM scheduling algorithm shows much balanced TPR results than the other two schedulers. Hence, it can be concluded to be a better energy-efficient algorithm than PF and BCQI scheduler.

\section{Conclusion}

This work mainly focuses on designing an efficient algorithm for LTE UL and DL, considering the constraints associated with them. The algorithm is expected to perform spectrally efficient under satisfactory level of fairness. The standard channel-aware scheduling algorithms were analyzed and compared against the proposed algorithms. These algorithms provide solution for the scheduling problem without satisfying the constraints associated with LTE UL and DL. Thus, there exists a need for new and improved scheduling strategy. Hence, the work mentioned in this thesis presents two distinctive scheduling algorithms for LTE UL and DL respectively. The proposed algorithm is compared against the standard scheduling schemes, namely PF and BCQI algorithms.

The uplink scheduling algorithm consists of two resource allocation matrices, primary matrix $\left(H_{1}\right)$ and secondary matrix $\left(H_{2}\right)$. The matrices combine the benefit of BCQI and PF algorithm such that, $H_{1}$ is throughput-centric and $H_{1}$ is fairness-centric. The primary matrix is initially employed to identify the UE-RB with highest metric and mapping that RB to the UE. The contiguity constraint is satisfied by considering the adjacent RBs of the previously allocated RB to the same user. The secondary matrix is employed additionally consider the contiguous allocation of the UE given that the previous loop on the primary matrix fails. Before assigning an RB to any UE, the power constraint is checked to ensure energy efficiency.

The downlink scheduling algorithm is an extension of the dual metric uplink scheduling algorithm with appropriate modification for LTE DL. Since the eNodeB is not as power-limited as the UE, avoiding power and contiguity constraints will result in better throughput performance. Similar to UL algorithm, the modified downlink algorithm consists of the primary and secondary resource allocation matrices. The algorithm consists of two phases, with primary matrix being used in the first phase and both primary and secondary matrices are used in the second phase. In the first phase the scheduler identifies the RB-UE with highest metric and map the selected RB to the UE, similar to the uplink algorithm. The second phase is used to extend the allocation for the user provided the fairness is not compromised. This is achieved by introducing a fairness constraint based on the resource allocation in the past subframes. The second level of the algorithm constructs a search-tree with two branch nodes evaluated for the intermittent RBs overruling contiguity constraint to the RB mapped. If any of the branch node satisfy a permissible level (Node score cut-off), then the corresponding RB is assigned to the user. 
The performance of the proposed algorithm is analyzed by comparing against standard scheduling algorithms. Assuming BCQI to give 100\% throughput performance, the proposed uplink scheduling algorithm achieves around 91\% to 94\% system throughput and PF algorithm achieves about $74.8 \%$ to $78 \%$ throughput performance. ODM uplink algorithm provides $32 \%$ to $35 \%$ more fairness when compared with BCQI algorithm and $17 \%$ to $19 \%$ less fairness than PF algorithm. In terms of transmit power, the proposed uplink algorithm outperforms PF and BCQI algorithm for the users with good and moderate channel conditions. Similarly, the proposed downlink algorithm produces better trade-off between throughput and fairness when compared against PF and BCQI algorithm. Assuming BCQI to give $100 \%$ throughput performance, ODM downlink algorithm shows $90 \%$ to $93 \%$ system throughput which is nearly $14 \%$ more than the performance of PF algorithm. In terms of fairness, the proposed scheme shows $11 \%$ to $18 \%$ less performance than PF and $36 \%$ to $45 \%$ more performance fairness than BCQI algorithm.

As an enhancement to this work, considering additional constraints such as delay requirement to the decision problem, the performance of the real time and non-real time services can be analyzed.

\section{References}

[1] 3GPP TS 36-Series, http://www.3gpp.org/LTE.

[2] 3GPP, "Proposed Study Item on Evolved UTRA and UTRAN," 3GPP TD RP-040461, Dec. 2004.

[3] 3GPP, "3rd Generation Partnership Project; Technical Specification Group Radio Access Network; E-UTRA; LTE Physical layer; General description (Rel. 10)," 3GPP TS 36.201 V10.0, Dec. 2010.

[4] White Paper, "Long Term Evolution Protocol Overview," Freescale Semiconductor, 2008. [Online] http://www.freescale.com/.

[5] S. Sesia, I. Toufik and M. Baker, "LTE - The UMTS Long Term Evolution from Theory to Practice,” John Wiley \& Sons Publication, 2011, pp. 87-120.

[6] H. Myung, J. Lim and D. Goodman, "Single carrier FDMA for uplink wireless transmission," IEEE Vehicular Technology Magazine, pp. 30 - 38, Sept. 2006. http://dx.doi.org/10.1109/MVT.2006.307304

[7] A. Kanagasabai and A. Nayak, "Opportunistic Dual Metric Scheduling Algorithm for LTE Uplink", IEEE Intl. Workshop on Smart Communication Protocols and Algorithms, 2015. http://dx.doi.org/10.1109/ICCW.2015.7247382

[8] S. Schwarz, J. Ikuno, M. Simko, M. Taranetz, Q. Wang and M. Rupp, "Pushing the 
Limits of LTE: A Survey on Research Enhancing the Standard," in IEEE Access, 2011. http://dx.doi.org/10.1109/ACCESS.2013.2260371

[9] J. Blumenstein, J. Ikuno, J. Prokopec and M. Rupp, "Simulating the Long Term Evolution Uplink Physical Layer," in Proc. of the 53rd Int. Symp. ELMAR, Zadar, Croatia, Sept. 2011

[10] "MATLAB”,MathWorks, http://www.mathworks.com/products/matlab.

[11] A. Gessner, A. Roessler and M. Kottkamp, "UMTS Long Term Evolution (LTE) Technology Introduction," White Paper, Rohde Schwarz, July 2012.

[12] B. Tirouvengadam, R. Radhakrishnan and A. Nayak, "CAAHR: Content Aware Adaptive HARQ Retransmission Scheme for 4G/LTE Network," IEEE Int. Conf. on Ubiquitous and Future Networks, pp. 456-461, 2012. http://dx.doi.org/10.1109/ICUFN.2012.6261749

[13] 3GPP, "3rd Generation Partnership Project; Technical Specification Group Radio Access Network; MAC Protocol Specification (Rel. 5)”, 3GPP TS 25.321 V5.10.0, Dec. 2004.

[14] F. Capozzi, G. Piro and et al., "Downlink Packet Scheduling in LTE Cellular Networks: Key Design Issues and a Survey," IEEE Communications Surveys and Tutorials, vol. 15, pp. 678 - 700, 2013. http://dx.doi.org/10.1109/SURV.2012.060912.00100

[15] A. Baid, R. Madan and A. Sampath, "Delay Estimation and Fast Iterative Scheduling Policies for LTE Uplink," Int. Symp. on Modeling and Optimization in Mobile, Ad Hoc and Wireless Networks, pp. 89 - 96, 2012.

[16] F. Ghandour et al, "A Fair and Power Saving Uplink Scheduling Scheme for 3GPP LTE Systems," Int. Conf. on the Network of the Future, pp. 6-9, 2011. http://dx.doi.org/10.1109/NOF.2011.6126686

[17] H. Haas and S. Videv, "Energy-Efficient Scheduling and Bandwidth-Energy Efficiency Trade-Off with Low Load," IEEE Int. Conf. on Communications, pp. 1-4, 2011. http://dx.doi.org/ 10.1109/icc.2011.5962571

[18] G. Piro, L. A. Grieco and et al, "Two-Level Downlink Scheduling for Real-Time Multimedia Services in LTE Networks", IEEE Transaction on Multimedia, vol. 13, pp. 1052-1065, 2011. http://dx.doi.org/10.1109/TMM.2011.2152381

[19] A. Reyhani Masoleh, "Resource Allocation in Uplink Long Term Evolution", M.S. Thesis, Univ. of Western Ontario, 2013.

[20] E. Yaacoub and Z. Dawy, "A Game Theoretical Formulation for Proportional Fairness in LTE Uplink Scheduling," IEEE Conf. on Wireless Communications and Networking, pp. 1-5, 2009. http://dx.doi.org/10.1109/WCNC.2009.4917504

[21] F. Calabrese, et al, "Search-Tree Based Uplink Channel Aware Packet Scheduling for 
UTRAN LTE," IEEE Vehicular Technology Conference, pp. 1949 - 1953, 2008. http://dx.doi.org/10.1109/VETECS.2008.441

[22] Chiapin Wang, et al, "Hybrid maximum-rate and proportional-fairness resource allocation in the downlink of LTE networks," Int. Conf. on Consumer Electronics, pp. 23 - 24, 2014. http://dx.doi.org/10.1109/ICCE-TW.2014.6904073

[23] R. K. Jain, D. W. Chiu and W. R. Hwae, "A Quantitative Measure of Fairness and Discrimination for Resource Allocation in Shared Computer Systems," DEC Research, Tech. Rep. 301, 1984.

[24] 3GPP, "LTE; Evolved Universal Terrestrial Radio Access (E-UTRA); User Equipment radio transmission and reception," 3GPP TS 136101 V10.3.0, 2011.

[25] M. Lauridsen, A. Jensen and P. Mogensen, "Reducing LTE Uplink Transmission Energy by Allocating Resources," IEEE Vehicular Technology Conference, pp. 1-5, 2011. http://dx.doi.org/10.1109/VETECF.2011.6092935

[26] A. Brunstrom, T. Ottosson and K. Norlund, "Fairness measures for best effort traffic in wireless networks," IEEE Int. Symp. on Personal, Indoor and Mobile Radio Communications, vol. 4, pp. 2953 - 2957, 2004.

http://dx.doi.org/10.1109/PIMRC.2004.1368861

[27] B. Tirouvengadam and A. Nayak, "Hybrid Mode Radio Link Control for Efficient Video

Transmission over 4G LTE Network," IEEE 18th Intl. Conf. on Parallel and Distributed Systems, pp. 22-29, 2012. http://dx.doi.org/10.1109/ICPADS.2012.14

[28] R. Radhakrishnan, B. Tirovengadam and A. Nayak, "Cross Layer Design for Efficient

Video Streaming over LTE Using Scalable Video Coding," Network Protocols and Algorithms, Vol 4, No 4, pp. 101-125, 2012. http://dx.doi.org/10.1109/ICC.2012.6364725

\section{Copyright Disclaimer}

Copyright reserved by the author(s).

This article is an open-access article distributed under the terms and conditions of the Creative Commons Attribution license (http://creativecommons.org/licenses/by/3.0/). 